Retraction Note: Effect of perioperative infusion of Dexmedetomidine combined with Sufentanil on quality of postoperative analgesia in patients undergoing laparoscopic nephrectomy: a CONSORTprospective, randomized, controlled trial

Fuxi Song ${ }^{1}$, Chunmiao Ye ${ }^{2}$, Feng Qi ${ }^{3}$, Ping Zhang ${ }^{2}$, Xuexiang Wang ${ }^{4}$, Yanfeng Lü ${ }^{5}$, Alejandro Fernandez-Escobar ${ }^{6}$, Chao Zheng ${ }^{2^{*}}$ and Liang $\mathrm{Li}^{2^{*}}$

Retraction Note: BMC Anesthesiol (2018) 18:145

https://doi.org/10.1186/s12871-018-0608-3

The authors have retracted this article [1] because they have recently found that the clinical results for the dosage of postoperative opioids in some patients were inconsistent with the results presented. In light of these findings, the authors no longer have confidence in the accuracy of the reported data and the conclusions of the article and wish to redesign the programme of postoperative analgesia and re-analyse their work. The authors would like to apologise for any inconvenience. All authors agree to this retraction.

\section{Author details}

'Department of Anaesthesiology, the Second Hospital of Shandong University, No. 247 Beiyuan Street, Jinan 250033, China. ${ }^{2}$ Department of Breast Surgery, the Second Hospital of Shandong University, No. 247 Beiyuan Street, Jinan 250033, China. ${ }^{3}$ Department of Anaesthesiology, Qilu Hospital of Shandong University, No. 107 Wenhua West Road, Jinan 250012, China. ${ }^{4}$ The Institute for Translational Medicine, the Second Hospital of Shandong University, No. 247 Beiyuan Street, Jinan 250033, China. ${ }^{5}$ Department of Anoproctology, the Second Hospital of Shandong University, No. 247 Beiyuan Street, Jinan 250033, China. ${ }^{6}$ Translational Research Program, University of Toronto, 27 King's College Circle, Toronto, ON M5S 1A1, Canada.
Received: 25 January 2019 Accepted: 25 January 2019

Published online: 13 February 2019

Reference

1. Song, et al. Effect of perioperative infusion of Dexmedetomidine combined with Sufentanil on quality of postoperative analgesia in patients undergoing laparoscopic nephrectomy: a CONSORT-prospective, randomized, controlled trial. BMC Anesthesiol. 2018;18:145. https://doi.org/10.1186/s12871-018-0608-3.

* Correspondence: chaozheng@sdu.edu.cn; liliang@sdu.edu.cn

2Department of Breast Surgery, the Second Hospital of Shandong University, No. 247 Beiyuan Street, Jinan 250033, China

Full list of author information is available at the end of the article

(c) The Author(s). 2019 Open Access This article is distributed under the terms of the Creative Commons Attribution 4.0 International License (http://creativecommons.org/licenses/by/4.0/), which permits unrestricted use, distribution, and reproduction in any medium, provided you give appropriate credit to the original author(s) and the source, provide a link to the Creative Commons license, and indicate if changes were made. The Creative Commons Public Domain Dedication waiver (http://creativecommons.org/publicdomain/zero/1.0/) applies to the data made available in this article, unless otherwise stated. 